\title{
Enjeux et transformations des régions métropolitaines du sud du Rhin supérieur
}

Challenges and transformations of metropolitan areas in southern Upper Rhine Architexturen zukunftsfähiger Metropolitanregionen am Südlichen Oberrhein

\section{Rita Schneider-Sliwa}

\section{grevues.org}

Édition électronique

URL : http://rge.revues.org/1718

ISSN : 2108-6478

\section{Éditeur}

Association des géographes de l'Est

Édition imprimée

Date de publication : 1 septembre 2008

ISSN : 035-3213

\section{Référence électronique}

Rita Schneider-Sliwa, «Enjeux et transformations des régions métropolitaines du sud du Rhin supérieur ", Revue Géographique de l'Est [En ligne], vol. 48 / 3-4 | 2008, mis en ligne le 02 mars 2010 consulté le 30 septembre 2016. URL : http://rge.revues.org/1718

Ce document a été généré automatiquement le 30 septembre 2016.

Tous droits réservés 


\title{
Enjeux et transformations des régions métropolitaines du sud du Rhin supérieur
}

Challenges and transformations of metropolitan areas in southern Upper Rhine Architexturen zukunftsfähiger Metropolitanregionen am Südlichen Oberrhein

\author{
Rita Schneider-Sliwa
}

\section{NOTE DE L'ÉDITEUR}

Texte déjà publié dans : Haase, F. et E. Jacob (éds). - Architexturen einer Grenzregion. Medien und Kommunikation am Oberrhein, Munich, Koepad (avec le soutien du Secrétariat d'Etat à l'économie SECO).

\section{Introduction}

1 Dans une «Europe des régions » aux frontières mobiles, des relations institutionnelles et de longues années de travail préparatoire destiné à la coopération transfrontalière offrent plus que jamais des possibilités de renouvellement en matière de communication et de planification des régions métropolitaines transfrontalières. La recherche de nouveaux modèles permettant d'envisager l'avenir durable de régions urbaines compétitives nécessite cependant de connaître plus précisément les formes d'organisation des régions métropolitaines. Il importe également de s'inspirer de certaines bonnes pratiques et de savoir dans quelle mesure le développement durable des régions métropolitaines peut être géré et quels sont les cadres institutionnels qui sont nécessaires pour y parvenir. 
2 Dans ce contexte, cet article présente différentes approches institutionnelles pratiquées actuellement en matière de développement des régions métropolitaines dans les espaces métropolitains de Strasbourg et de Bâle. L'objectif est de proposer une réflexion sur la politique de développement urbain de la région métropolitaine transfrontalière de Bâle, tout en prenant en compte les différents modèles d'organisation, les structures régionales de gestion et les formes juridiques. Ceci permettra de montrer dans quelle mesure ces modèles peuvent intéresser la politique urbaine de la région métropolitaine transfrontalière de Bâle. L'article pose notamment la question de savoir si le cadre de l'Eurodistrict de Bâle est suffisant pour assurer l'avenir de la région métropolitaine transfrontalière. Le cas de Strasbourg-Ortenau permet de montrer qu'il existe une région métropole transfrontalière opérationnelle aux portes de Bâle.

\section{Les défis du développement des régions métropolitaines du sud du Rhin supérieur}

\section{A. Défis politiques}

3 Dans une "Europe des régions", les frontières politiques demeurent des lignes de séparation entre différentes normes juridiques, différentes cultures de planification et différents styles et standards de vie. Le développement économique des régions urbaines constitue toujours un défi pour les Etats, les cantons et les communes frontalières. Il nécessite de tenir compte des cultures particulières qui interviennent dans la politique de planification et d'aménagement transfrontalière et nécessite, parfois, de faire preuve de courage pour proposer de nouvelles options. Depuis plus de quarante ans, les institutions et les organes des trois pays riverains du Rhin supérieur travaillent au développement de la région (voir Speiser 1993; http://www.regbas.ch/f_datescle.cfm; http:// www.regiotrirhena.org/dn_situation_region), chaque décennie apportant son lot de nouveaux organismes et de nouvelles institutions. Du point de vue scientifique, la multiplication des efforts et des formes institutionnelles de la coopération obtenue à chaque décennie peut être considérée comme une accumulation de paradigmes sur la question de la planification optimale des régions métropolitaines transfrontalières. Ces efforts reflètent l'état actuel de la connaissance et des compétences et sont dignes d'intérêt, comme en témoignent certaines études conduites sur le sujet (Minghi 1977; Gallusser 1994; Newman et Paasi 1998; Eder et Sandtner 2000; Schneider-Sliwa 2003; Fichtner 2006; Gschwind 2003). La recherche des formes de coopération transfrontalière les plus adéquates est cependant loin d'être achevée dans le Rhin supérieur. Même les nouveaux eurodistricts ne peuvent être considérés comme des modèles aboutis en matière d'organisation de la coopération transfrontalière, mais vont sans doute évoluer dans le futur.

\section{B. L'espace économique du sud du Rhin supérieur}

4 Le potentiel économique du sud du Rhin supérieur est remarquable (figure 1): 700 millions d'Européens de l'Est et de l'Ouest peuvent être facilement atteints depuis cette région. La proximité géographique de plusieurs marchés européens - l'un au fort pouvoir d'achat à l'Ouest et l'autre en forte croissance et bénéficiant d'un grand potentiel de rattrapage à l'Est - est favorable à des relations d'affaires (Chambre de Commerce 
Baselland 2004). Le sud du Rhin supérieur constitue une plate-forme à la fois continentale et intercontinentale pour le transport de fret, reliée aux quatre océans (figure 2). Grâce à des trains à grande vitesse, à des compagnies aériennes à prix réduits et à des transporteurs routiers rapides, la quasi-totalité de l'espace économique européen se trouve dans l'aire d'attraction des localités du sud du Rhin supérieur. Cet espace économique possède une structure urbaine équilibrée, composée des régions métropolitaines de Bâle, Strasbourg et Karlsruhe, qui comptent chacune près d'un million d'habitants (figure 3).

Figure 1 : Pôles de croissance européens

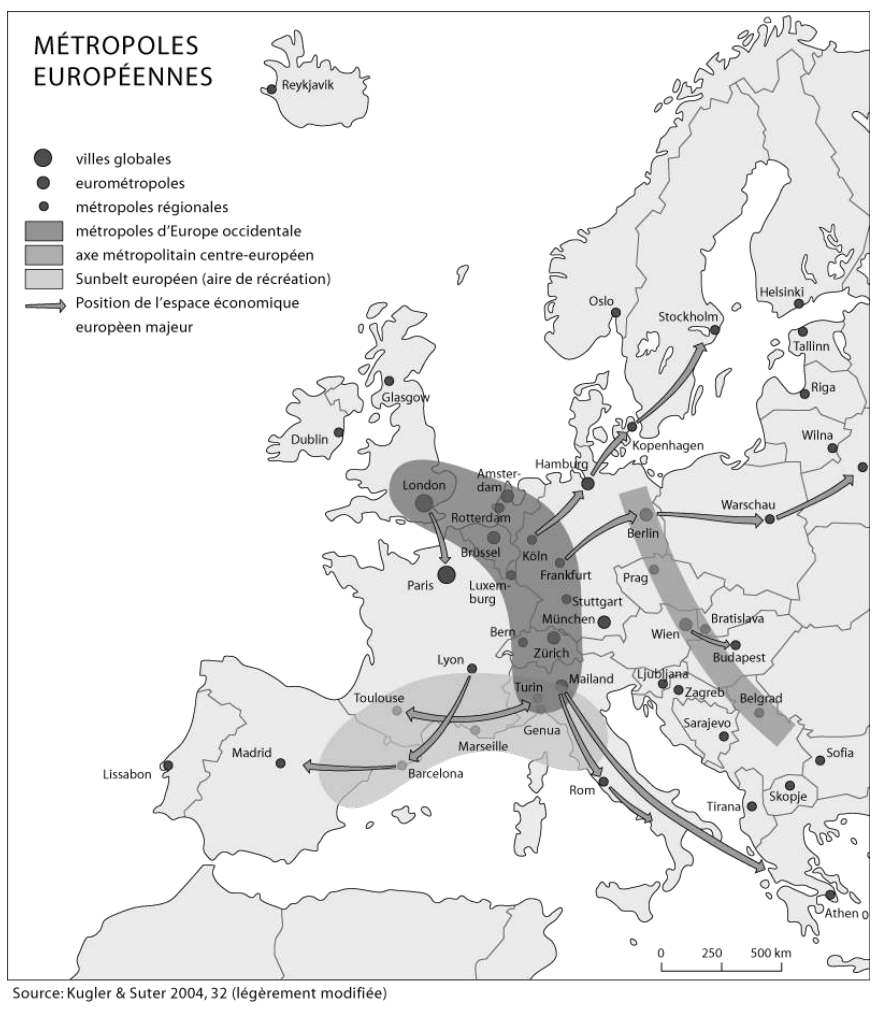

Cartographie : Leena Baumann 
Figure 2 : Carrefours européens du transport de marchandises et du fret maritime

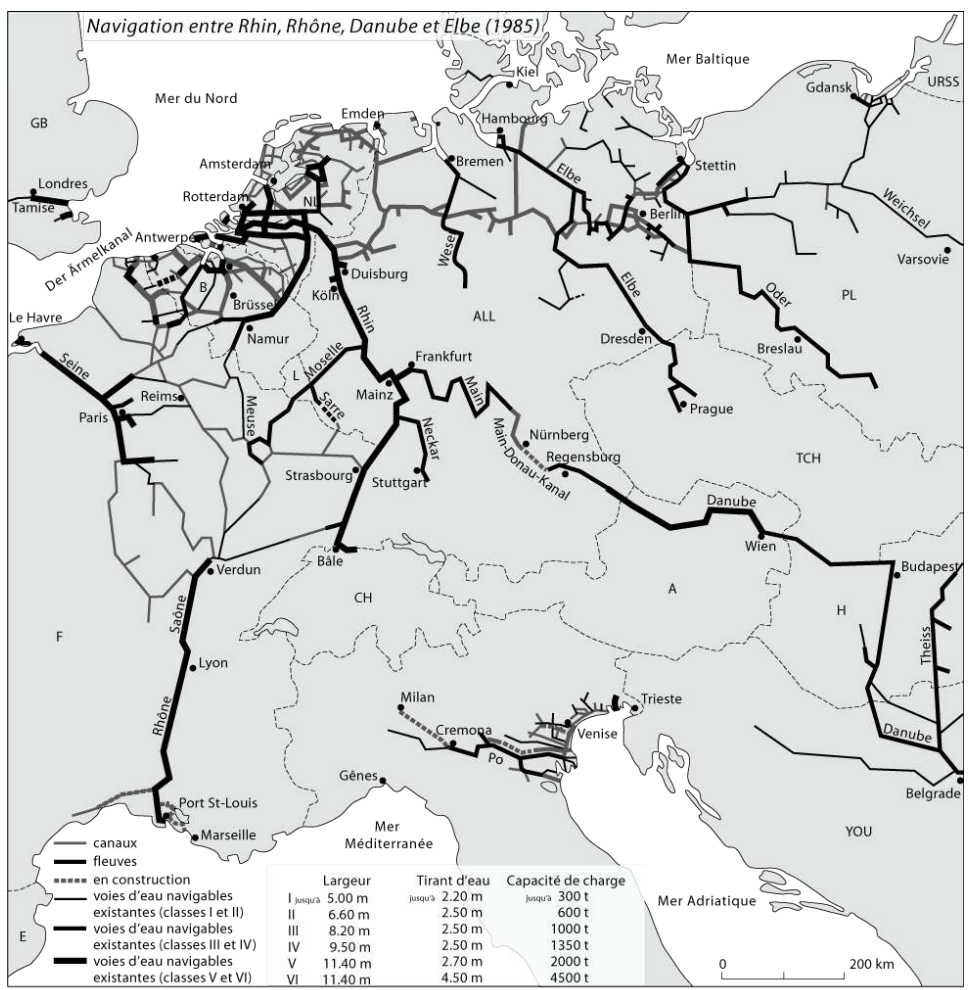

Cartographie : Leena Baumann

Figure 3 : Région de Metrobasel

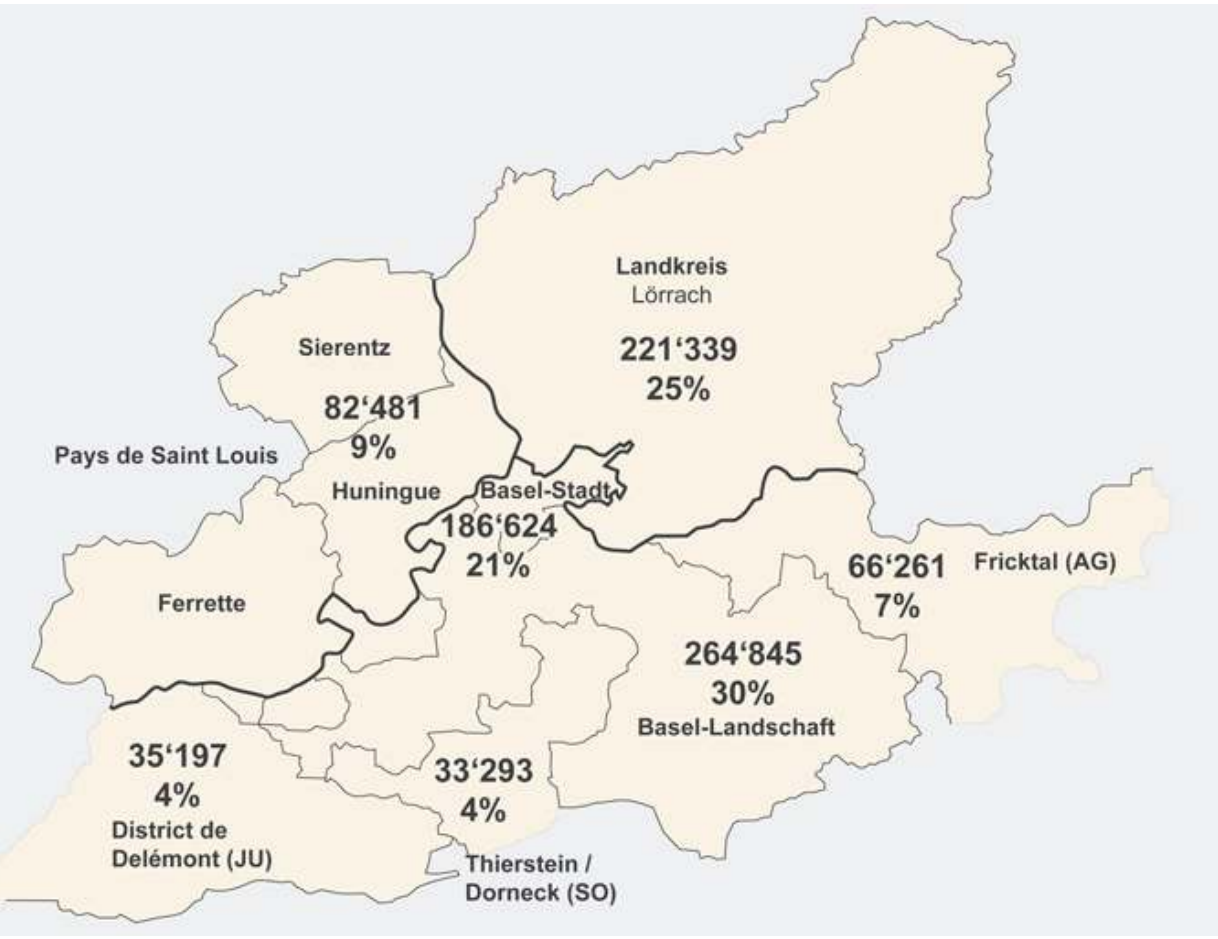

Cartographie : Leena Baumann 
5 Ainsi, la région offre une palette presque complète d'industries et de services existants, un potentiel favorable à l'implantation de nouvelles activités commerciales et de services ainsi que des réserves foncières dans la partie allemande et française. Au point de vue international, l'Alsace, qui fait partie de la BioValley, attire des investisseurs dans le domaine de la biologie moléculaire, de la génétique, de la recherche supramoléculaire et des neurosciences (www.alsace-biovalley.com; www.biovalley.com; www.alsace.cnrs.fr ; www.inserm.fr). Dans la région de Karlsruhe, les hautes technologies et le développement du savoir constituent le moteur de l'économie. Dans la région de Bâle également, le cluster des sciences de la vie cherche à accroître sa renommée internationale. Le sud du Rhin supérieur dispose par conséquent de conditions favorables en tant que pôle économique.

\section{Les défis économiques de la région métropolitaine de Bâle}

6 Néanmoins, la région de Bâle n'a pas exploité toutes ses potentialités. La question du rôle des collectivités dans l'organisation et la gestion des régions métropolitaines y demeure urgente, notamment en ce qui concerne le développement économique. Le Rhin supérieur possède certes une longue expérience de coopération transfrontalière, mais il semble que, dans la région de Bâle, le monde politique et administratif déplore le fait que l'ensemble soit géré de manière peu rationnelle et efficace du côté suisse (Basler Zeitung, 3 janvier 2008, p. 19). La compétitivité au niveau européen ne peut être fondée que sur l'acquis de conditions favorables, tant il est également nécessaire de positionner la région métropolitaine de Bâle de manière proactive. A cet égard, la Regio TriRhena a encore un long chemin à parcourir du point de vue scientifique et administratif, un fait que les responsables politiques déplorent de plus en plus publiquement, en dépit de la mise en réseau de groupes, d'associations et d'institutions destinés à promouvoir la coopération transfrontalière :

7 «La Regio TriRhena (...) doit faire la preuve qu'elle peut exister et continuer à fonctionner entre l'Eurodistrict d'une part et une région métropolitaine trinationale du type de metrobasel d'autre part. Au niveau européen, il n'est pas encore décidé quelle sera la structure la plus appropriée à la poursuite du développement économique trinational du Rhin supérieur: une seule région métropolitaine contenant plusieurs centres ou deux régions métropolitaines, l'une au nord avec Strasbourg et Karlsruhe, l'autre au sud avec Bâle. Les dépenses relativement importantes occasionnées ainsi que la multiplicité des organismes impliqués poussent à revendiquer une rationalisation et une simplification des structures existantes » (Fichtner 2006, p. 102, notre traduction).

8 Alors que l'agglomération de Strasbourg-Ortenau sait mettre en valeur ses potentiels au moyen d'une planification exemplaire, la région de Bâle, qui bénéficie pourtant d'une certaine aisance matérielle, d'un taux de chômage faible et de sa situation en dehors de l'UE, a pu se baser plus longtemps sur des structures qui semblent apparemment avoir fait leurs preuves jusqu'à aujourd'hui.

\section{Un besoin d'adaptation rapide dans la région de Bâle et la Suisse du Nord-Ouest}

9 Outre les forces précédemment évoquées, il est bien évident que l'espace économique bâlois et son organisation sont également marqués par certaines faiblesses, mises en 
évidence depuis peu (Füeg 2006 : Wirtschaftsstudie Nordwestschweiz ; Regionalkonferenz der Regierungen der Nordwestschweiz 2005. Wirtschaftsraum Nordwestschweiz. Planerkonferenz der Regierungen der Nordwestschweiz 10 juin 2005 ; Basler Zeitung 3 janvier 2008). La région de Bâle a ainsi perdu 11808 emplois à plein temps entre 2001 et 2005 dans les domaines de l'industrie, de l'artisanat et des services, à l'exception de l'administration publique où une augmentation de 5357 emplois à plein temps a été observée (Füeg 2006). Dans ce contexte, il est également important de noter que, si la valeur ajoutée a augmenté pendant la même période, la réduction des effectifs a porté atteinte à la croissance économique régionale et au pouvoir d'achat. De même, les finances publiques ont été négativement affectées et doivent aujourd'hui répondre à une hausse du chômage et aux transferts financiers, au moins temporairement.

Du point de vue de la Conférence régionale des gouvernements de la Suisse du NordOuest, il ressort que la partie suisse du Rhin supérieur constitue un espace qui n'est pas épargné par les problèmes économiques et par une baisse de la compétitivité. Ceci va de pair avec une certaine rigidité institutionnelle, qualifiée de "petit esprit cantonal» ( Kantönligeist), qui n'exploite pas encore suffisamment les nouvelles possibilités de coopération. Pour appréhender les problèmes de la compétitivité régionale de manière proactive, amortir les effets des réductions du nombre de postes de travail ou créer de nouveaux emplois, il ne suffit donc plus de s'appuyer sur le climat économique accueillant, sur une régulation légère ou sur les avantages fiscaux qui sont caractéristiques de la Suisse (BAK Basel Economics 2005). En outre, les pôles économiques dynamiques ne peuvent jouer cavalier seul, en s'appuyant, comme les communes ou les cantons, sur la fixation des taux d'imposition. Ces pratiques anciennes, qui regagnent de la vigueur, conduisent à une concurrence interne entre les communes à l'intérieur des régions métropolitaines, alors que l'espace de vie et de travail de la métropole devrait bénéficier d'une émulation collective.

\section{Changement de paradigme : nouvelle forme d'organisation intercommunale et coopération régionale urbaine}

11 L'Accord de Karlsruhe de 1996 constitue une nouveauté juridique dont le potentiel n'est pas encore suffisamment reconnu et utilisé. Il offre des possibilités de coopération intercommunale allant au-delà des potentialités du système multi-niveaux du Rhin supérieur (figure 4). Cet Accord entre la Suisse, l'Allemagne, la France et le Luxembourg permet aux communes de chaque pays de construire des Groupements locaux de coopération transfrontalière (GLCT) bi ou trinationales, connus en Suisse sous le nom de regroupements de communes (Zweckgemeinden). Les GLCT peuvent conduire à des accords portant sur la souveraineté de la planification et permettent de disposer d'un budget spécifique pour la mise en œuvre de préoccupations communes. La coopération transfrontalière est possible dans les domaines suivants: développement économique transfrontalier, utilisation du sol et planification, infrastructures, aménagement du territoire et protection de l'environnement. 
Figure 4 : Le système multi-niveaux du Rhin supérieur

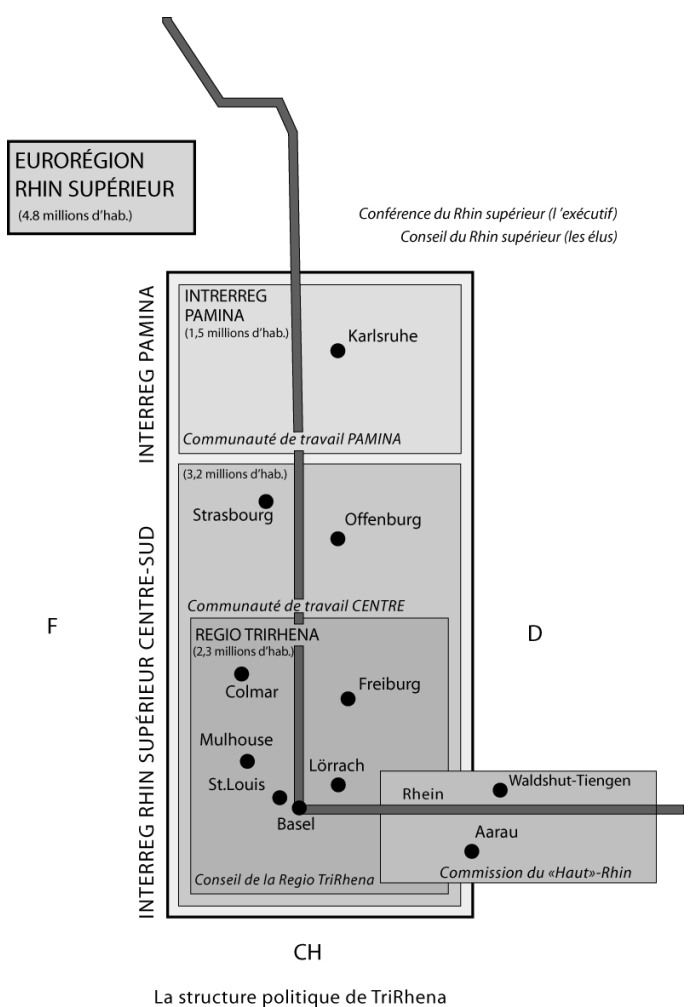

Cartographie : Leena Baumann

Les avantages offerts par les GLCT sont susceptibles renforcer les régions métropolitaines. Cette préoccupation est présente depuis 2001 dans la Politique des agglomérations de la Confédération suisse. L'Accord de Karlsruhe règle les obligations, les compétences en matière de planification et les aspects financiers des GLCT dans les articles 8, 10 et 11 (http://archiv.jura.uni-saarland.de/BIJUS/karlsruhe/gesetz.htm) :

\section{Article 8 Organismes de coopération transfrontalière}

(1) Les conventions de coopération transfrontalière peuvent prévoir la création d'organismes sans personnalité juridique (Article 9), la création d'organismes dotés d'une personnalité juridique ou la participation à ces organismes (Article 10), ou la création d'un groupement local de coopération transfrontalière (Article 11), de manière à prévoir la mise en cuvre efficace de la coopération transfrontalière.

Article 10 Organismes dotés de la personnalitéjuridique

Les collectivités territoriales ou organismes publics locaux peuvent participer à des organismes dotés de la personnalité juridique ou créer de tels organismes si ces derniers appartiennent à une catégorie d'organismes habilités dans le droit interne de la Partie où ils ont leur siège à comprendre des collectivités territoriales étrangères

Article 11 Groupement local de coopération transfrontalière

(1) Un groupement local de coopération transfrontalière peut être crée par les collectivités territoriales et organismes publics locaux en vue de réaliser des missions et des services qui présentent un intérêt pour chacun d'entre eux. Ce groupement local de coopération transfrontalière est soumis au droit interne applicable aux établissements publics de coopération intercommunale de la Partie où il a son siège. 
19 (2) Le groupement local de coopération transfrontalière est une personne morale de droit public. La personnalité juridique lui est reconnue à partir de la date de l'entrée en vigueur de la décision de création. Il est doté de la capacité juridique et de l'autonomie budgétaire.

L'Accord de Karlsruhe prévoit donc explicitement que les communes frontalières puissent instaurer des intercommunalités sans faire appel aux instances des Länder allemands, des cantons suisses ou des instances nationales afin de répondre à des tâches publiques.

21 Mais quels sont ces GLCT et que permettent-ils? Il s'agit de groupements de communes établis sur une base volontaire qui ne conduisent pas à une fusion communale. Leur objectif commun est la réalisation de tâches intercommunales bien définies. Les membres de ces intercommunalités peuvent intervenir en tant que collectivités territoriales possédant une personnalité morale et juridique. Les décisions sont prises par l'assemblée intercommunale, laquelle dispose d'une autonomie budgétaire en matière de planification, déléguée par chacun des membres de l'association. Ces GLCT possèdent les compétences et les finances nécessaires à la mise en œuvre effective des aménagements. Le rôle des associations de droit public (Zweckverbände) dans la planification intercommunale des régions métropolitaines est connu depuis le début du XXe siècle dans un grand nombre de pays européens. En Suisse, ces associations n'ont fait leur apparition dans le débat public que depuis peu (Frey et Eichenberger 1999 ; Kirchgessner, Feld et Savioz 1999 ; Saladin 1995 ; de Spindler 1998 ; Weber-Mandrin 2001).

\section{L'exemple de la région métropolitaine de Strasbourg}

L'Accord de Karlsruhe permet à la région métropolitaine de Bâle ou à l'Eurodistrict Trinational de Bâle de mettre en œuvre des structures de coopération intercommunale plus efficaces, qui possèdent des compétences et des obligations. Il est cependant utile de rappeler que l'existence seule d'un eurodistrict n'est pas suffisante pour parvenir à renforcer le rôle économique d'une région urbaine, comme le montre l'exemple de Strasbourg. Dans la région métropolitaine de Strasbourg, l'Eurodistrict s'appuie sur les intercommunalités possédant des structures juridiquement contraignantes et possédant des ressources fiscales propres. De cette façon, le potentiel d'un eurodistrict peut a priori être mieux exploité que dans une structure opérant en tant qu'association, mais sans réelle compétence juridique et financière, et qui reste donc non contraignant pour ses membres. Il vaut donc la peine de considérer la région métropolitaine de StrasbourgOrtenau de manière plus précise, étant donné qu'il s'agit d'un cas exemplaire situé dans le Rhin supérieur.

\section{A. Le Groupement Local de Coopération Transfrontalière : une structure centrale}

23 Avant que l'Eurodistrict ne soit créé, Strasbourg possédait une intercommunalité fonctionnelle, qui avait été organisée pour répondre aux défis du développement urbain transfrontalier. Cette communauté nommée « Syndicat mixte pour le schéma directeur de la région de Strasbourg » est composée de 11 communautés de communes de la Région Alsace et du Département du Bas-Rhin, dont la ville de Strasbourg et ses 27 communes 
périphériques (Communauté Urbaine de Strasbourg), de 6 communes isolées et de 139 communes appartenant à des syndicats communaux, et de 51 communes allemandes.

Ce Syndicat est chargé d'établir le schéma de développement de l'ensemble de la région urbaine de Strasbourg, connu sous le nom de "SCOTERS", qui signifie "Schéma de cohérence territoriale de la Région de Strasbourg » et créé en 1999. Le nom de SCOTERS est couramment utilisé comme synonyme du Syndicat en charge du schéma de développement de la région. Le SCOTERS appartient à une nouvelle forme de gouvernance urbaine, qui rend possible la mise en œuvre de nouvelles structures métropolitaines depuis 1966 et qui a trouvé un second souffle grâce aux lois françaises relatives à la décentralisation de 1982 et 1988. Depuis 1966, la France a décentralisé l'administration centrale dans les régions et les différents pouvoirs de décision au niveau local. Il s'agit notamment des organismes publics, indépendants de l'Etat et possédant une autonomie de gestion (figure 5). Au sein de la structure administrative française de dimension métropolitaine, les collectivités locales (municipalités) peuvent créer des associations de droit public, aux multiples finalités et fonctions.

Figure 5 : Cadre institutionnel relatif à la planification des régions métropolitaines en France

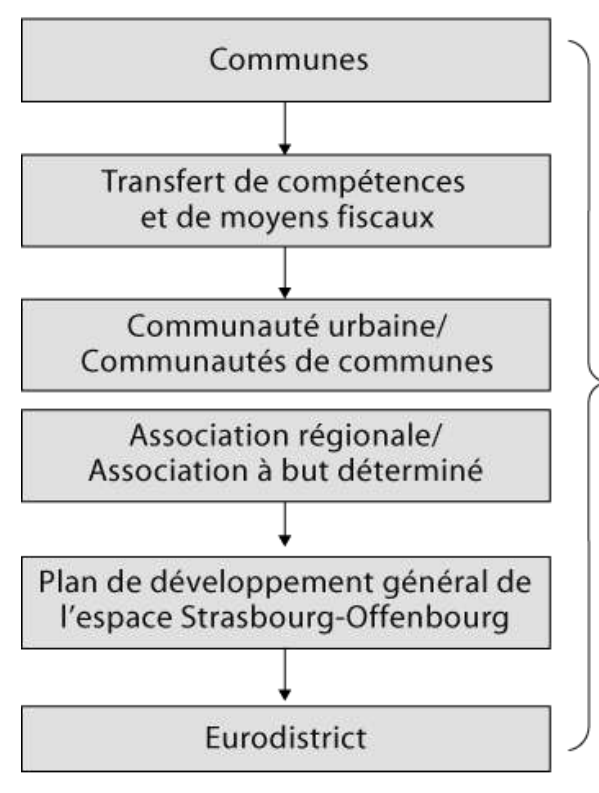

Plan de développement général:

Aménagement intersectoriel cohérent de l'espace économique Strasbourg-Ortenau en matière de:

- développement économique

- d'aménagement du territoire

Politique économique élaborée

pour l'espace Strasbourg-Ortenau:

- politique concertée de développement

économique pour l'ensemle du

territoire considéré

- politique infrastructurelle, focalisation sur

l'economie du savoir, les universités en tant que facteurs de développement local, mise en réseaux de l'enseignement supérieur

Aménagement du territoire:

- I'usage du sol et son management

- politique d'aménagement global des

zones d'activités

- parcs d'activités et du savoir:

aménagement global des lieux

\section{B. Souveraineté financière et compétences}

L'élément central dans l'architecture de la région métropolitaine de Strasbourg est le fait que les municipalités ont cédé une partie de leurs compétences aux Communautés Urbaines. Ces dernières se sont de leur part à nouveau regroupées au sein du Syndicat précédemment évoqué. Le plan d'aménagement de la région urbaine sera élaboré par le SCOTERS au travers d'un processus participatif et démocratique avec les communes, lesquelles ont intégré le Syndicat de façon volontaire mais contractuelle. Ces communautés transfèrent une partie de leurs recettes fiscales au Syndicat, lequel reçoit en outre un financement public pour la planification régionale de la part du gouvernement français. La base du financement du Syndicat est constituée par les 
ressources fiscales, qui proviennent à plus de $50 \%$ de la ville de Strasbourg elle-même et à $25 \%$ de la CUS. Il s'agit de la taxe d'habitation, à laquelle sont astreints les locataires, les propriétaires ainsi que les personnes physiques et morales, de la taxe foncière sur les propriétés bâties et non bâties, et de la taxe professionnelle. Il existe en outre d'autres possibilités de financement comme l'émission d'obligations municipales ou de certaines redevances relatives aux services urbains. Afin de favoriser la création d'entreprises, le SCOTERS se réserve également le droit de procéder à des exonérations fiscales.

\section{Les tâches de la planification}

Dans le domaine de l'aménagement du territoire, le Syndicat est en charge des tâches relatives au Master Plan, à la planification sectorielle, à l'occupation du sol, à l'urbanisme et s'occupe également de la planification des infrastructures et des transports. Dans le domaine du développement économique, le SCOTERS traite de la création, de la planification et de la gestion des surfaces concernant les activités industrielles, commerciales et de services, l'artisanat, les activités touristiques, le développement aéroportuaire et portuaire, ainsi que les parcs industriels et scientifiques. Le SCOTERS conçoit et réalise la politique de développement économique, y compris ce qui relève des infrastructures physiques.

La politique de développement économique dans la région métropolitaine de Strasbourg comprend la planification générale, la planification spécialisée et multi-usages, la politique du marché de l'emploi et de la formation, ainsi que l'élargissement des infrastructures. Un accent particulier est porté aux activités à forte intensité de connaissance et à la promotion du savoir et des clusters. Il est vrai qu'une dynamique similaire existe également dans la région métropolitaine de Bâle, comme en atteste le financement que certains acteurs privés apportent au Campus du Savoir, aux sites de recherche liés aux sciences de la vie ainsi qu'à l'Université de Bâle, qui manque singulièrement de moyens. Cependant, il est remarquable de constater que, dans la région métropolitaine de Strasbourg, le Syndicat précité ainsi que les acteurs étatiques agissent selon une planification concertée, afin de créer un environnement propice au savoir. L'approche de Strasbourg, au travers d'une planification globale s'appuyant sur un GLCT transfrontalier, est donc fondamentalement différente du modèle bâlois, fondé sur un type d'aménagement par opportunité (planning by opportunities), poursuivi également dans la nouvelle structure de l'Eurodistrict de Bâle (voir chap. v).

Un nombre impressionnant d'institutions se sont engagées dans une coopération contraignante avec le SCOTERS relativement au développement à long terme de la région métropolitaine de Strasbourg. En France, outre le SCOTERS et la CUS, le gouvernement de la République française, la Région Alsace et le Département du Bas-Rhin sont représentés. En Allemagne, le Kreis d'Ortenau, la Regionalverband du sud du Rhin supérieur, la ville d'Offenbourg ainsi que les villes de Kehl, Lahr et Achern sont également impliquées.

\section{Conclusion intermédiaire}

La planification binationale de la région métropolitaine de Strasbourg-Ortenau est caractérisée par la création d'un Syndicat fondé sur un partenariat stratégique et contraignant. Ce Syndicat doit répondre à ses obligations, dispose d'une autonomie financière et bénéficie de compétences en matière de planification. La coordination entre 
les gouvernements aux échelles binationales, locales, régionales et intercommunales est une condition du succès de cette structure. Le système de planification de la région métropolitaine de Strasbourg-Ortenau poursuit ainsi une approche intégrative par le bas (bottom up). Il s'agit d'un réseau polycentrique, international et orienté sur l'action, à l'intérieur duquel les compétences de toutes les communes concernées forment un ensemble cohérent.

Cette planification globale sert de fondement à la mise en place d'un Eurodistrict fonctionnel entre Strasbourg et Ortenau. La mise en place de cet Eurodistrict bénéficie par conséquent d'une longue histoire de planification, qui se caractérise par le fait que les communes participantes ont délégué certaines compétences financières et de planification à une structure commune. Même si certaines faiblesses demeurent, par exemple dans le développement social de la région de Strasbourg, et que le système de planification est en constante évolution, cette forme de gouvernance urbaine pourrait constituer un modèle d'avenir pour les régions métropolitaines, sans nécessairement se limiter au sud du Rhin supérieur.

\section{Comparaison avec la région métropolitaine de Bâle}

\section{A. Cadres structurels et Politique des agglomérations de la Confédération suisse}

31 Il est temps de considérer, dans une perspective comparatiste, la région métropolitaine de Bâle dans son contexte suisse. La Suisse compte environ 7,5 millions d'habitants divisés en près de 2800 communes, lesquelles appartiennent à 26 cantons différents qui possèdent chacun une administration spécifique. Il existe donc 26 plans directeurs cantonaux différents et 33 lois sectorielles de planification qui sont susceptibles de réglementer l'aménagement du territoire, y compris en milieu urbain. Ce contexte spécifiquement suisse oblige cependant de procéder à une certaine coordination des communes et des cantons, avec 22 conférences régionales et communales, 500 conférences de fonctionnaires, 311 accords cantonaux, 444 accords entre cantons et approximativement 1000 accords entre communes, ces derniers servant essentiellement des objectifs précis, comme la protection contre les incendies (Verein Metropole Schweiz 2003).

Pour faire face à cette diversité de manière plus efficace, renforcer les synergies et promouvoir certaines préoccupations communes aux villes et aux agglomérations, la Confédération suisse a décidé de mettre en œuvre une "Politique des agglomérations ». Cette politique conçue au plan national en 2001 s'adresse aux villes et à leurs communes environnantes. Afin d'utiliser plus judicieusement le potentiel de coopération et de l'ancrer plus fortement dans les consciences, la Conférence des gouvernements cantonaux (CdC) a donné naissance en 2011 à la Conférence tripartite sur les agglomérations (CTA), qui constitue une plate-forme commune à la Confédération, aux cantons, aux villes et aux communes.

Ainsi que le rappelle la Conférence des gouvernements cantonaux :

"Sur proposition de la $\mathrm{CdC}$, la Confédération, les cantons ainsi que les villes et les communes ont fondé en février 2001 la Conférence tripartite sur les agglomérations (CTA), qui a pour objectif de promouvoir la collaboration verticale Confédération - 
cantons - communes et villes ainsi que le développement d'une politique commune des agglomérations. La responsabilité de la CTA incombe aux cantons du fait de leur compétence constitutionnelle à l'égard des communes et des villes. C'est donc la $\mathrm{CdC}$ qui assume le secrétariat de la CTA. La CTA est une plate-forme commune de la Confédération, des cantons, des villes et des communes. La CTA vise une collaboration plus étroite entre la Confédération, les cantons, les villes et les communes ainsi que le développement d'une politique des agglomérations commune. Il s'agit, par le biais d'efforts coordonnés, d'améliorer les chances d'avenir des agglomérations en tant que catalyseurs du développement économique, social et culturel. Grâce à la CTA, il existe pour la première fois un organe de collaboration partenariale entre la Confédération, les cantons et les communes » (Conférence des gouvernements cantonaux 2007).

Grâce à 25 projets pilotes, la Confédération entend renforcer l'attractivité économique des villes et améliorer la qualité de vie de ses habitants. Elle souhaite également encourager un fonctionnement polycentrique des centres de rangs différents et possédant des fonctions complémentaires et entend appuyer le développement interne des agglomérations. Du point de vue de la Confédération suisse :

«La collaboration au sein des agglomérations doit être améliorée. Une collaboration existe déjà entre les communes, au travers d'institutions et sous les formes juridiques les plus diverses, mais elle a le plus souvent une vocation sectorielle et couvre des périmètres très variables. Les problèmes doivent être abordés d'un point de vue global. Les différentes politiques sectorielles, notamment l'urbanisation et les transports, doivent être mieux coordonnées" (SSSA 2002, voir http://www.sgvw.ch/f/focus/ Pages/021014_agglomeration_tobler.aspx, G. Tobler, La politique des agglomérations en Suisse, 14 octobre 2002).

\section{B. L'Agglomération Trinationale de Bâle, l'Eurodistrict Trinational de Bâle et metrobasel}

37 Avec l'Agglomération Trinationale de Bâle (ATB) fondée en 1995, l'agglomération de Bâle a fait figure de pionnier en matière de coopération transfrontalière aux niveaux cantonaux, intercommunaux et internationaux, bien avant l'avènement de la Politique des agglomérations de la Confédération suisse. L'Agglomération Trinationale de Bâle est cependant loin d'avoir atteint un optimum en termes de planification du fait que cette association n'est pas juridiquement contraignante, contrairement aux exemples allemands et français.

38 Le gouvernement cantonal de Bâle-Ville, pour lequel Bâle constitue le moteur de l'économie trinationale, a développé une certaine vision de la manière dont la planification métropolitaine doit être organisée institutionnellement. Selon cette vision, chaque acteur a une tâche bien précise: l'Etat (la Confédération) développe l'espace métropolitain en collaboration avec l'ATB et l'Eurodistrict, les acteurs du monde économique et scientifique effectuent quant à eux le monitoring périodique du développement de la région au moyen du « Metrobasel-Monitor», tandis que la société civile s'engage dans le projet metrobasel, qui doit conduire à ancrer la prise de conscience d'un espace partagé et d'intérêts communs. Cette dernière tâche est également assignée aux médias. Dans cette vision, les compétences entre ces domaines, considérés comme égaux, ne doivent pas être mélangées. Certes, la planification devrait être partagée entre les acteurs situés de part et d'autre des frontières nationales; cependant, la mise en 
œuvre des projets doit être effectuée séparément, avec l'aide des instances nationales (Lezzi M., cheffe de la Division principale de la planification du Département des constructions du canton de Bâle-Ville 2006, voir http://www.srl.de).

Le développement de la région métropolitaine trinationale de Bâle est ainsi marqué par deux initiatives: d'une part l'Eurodistrict Trinational de Bâle (ETB), d'autre part l'initiative metrobasel. Contrairement à d'autres villes et régions métropolitaines du sud du Rhin supérieur, Bâle s'appuie toujours sur une mise en œuvre séparée selon les espaces nationaux, sur une séparation des domaines d'intervention et sur des structures qui ne sont contraignantes ni financièrement ni juridiquement.

\section{Conclusion intermédiaire}

40 L'Eurodistrict Trinational de Bâle constitue un acteur important de la coopération transfrontalière. Cependant, en tant qu'association, il lui manque la forme juridique qui permette une véritable souveraineté en matière de planification ainsi que des compétences financières. En outre, l'ETB n'est pas encore une association fonctionnelle de communes urbaines et périurbaines comparable aux Communautés Urbaines de France par exemple. De même, l'ETB ne contient pas de syndicat qui pourraient aspirer à un tel développement.

De même, l'initiative metrobasel, qui a pour objectif de susciter une nouvelle prise de conscience de la région métropolitaine trinationale dans la société civile - sur le modèle du branding - ne possède pas le statut juridique, la souveraineté en matière de planification et les compétences financières correspondants. Par conséquent, ni l'Eurodistrict Trinational de Bâle ni l'initiative metrobasel, dans leur forme actuelle, ne peuvent constituer en soi un concept à long terme de développement durable pour la région métropolitaine. Le modèle bâlois fondé sur la planification par opportunités déjà évoqué, établi sans une forte institutionnalisation et sans compétences juridiques et financières, pourrait se révéler désavantageux à long terme. Le risque est d'autant plus élevé que les espaces voisins du Rhin supérieur conduisent des initiatives de planification fondées sur des institutions fonctionnelles, susceptibles de renforcer leur position dans la compétition des villes européennes et d'offrir une meilleure qualité de vie.

Jusqu'ici, le débat public portant sur les formes d'organisation spatiales de l'espace bâlois reste malheureusement très connoté politiquement, ce qui semble préjudiciable à l'analyse des questions fondamentales. Les nouvelles formes de coopération basées sur les intercommunalités sont ainsi souvent assimilées à des fusions de communes (Basler Zeitung 3 janvier 2008). De ce fait, l'importance et l'urgence des questions liées à un aménagement urbain et régional plus efficace fondé sur des associations communales semble trouver peu d'écho dans l'opinion avant même d'avoir une sérieuse chance d'être compris.

\section{Conclusion}

Alors que la région métropolitaine de Strasbourg a orchestré son développement au moyen d'intercommunalités de droit public possédant un statut de quasi-autonomie du point de vue de la planification et des ressources financières et permettant des actions durables, Bâle s'est appuyée encore jusqu'à tout récemment sur une planification par 
opportunités. Dans le modèle bâlois en effet, les discussions concertées entreprises entre les partenaires suisses, français et allemands ne sont pas contraignantes. En outre, les décisions auxquelles aboutissent ces discussions sont mises en œuvre séparément sur le principe de la souveraineté nationale même si les décisions sont prises en partie déjà en commun (figure 6). Afin de renforcer les régions métropolitaines du sud du Rhin supérieur, les aspects conceptuels liés à la planification et aux frontières politiques doivent être repensés. C'est à cette condition que les potentialités de la région métropolitaine de Bâle pourront assurer un avenir durable aux populations qui habitent l'espace transfrontalier.

Figure 6 : Des amorces différentes en matière de développement d'espaces économiques sur le Rhin supérieur

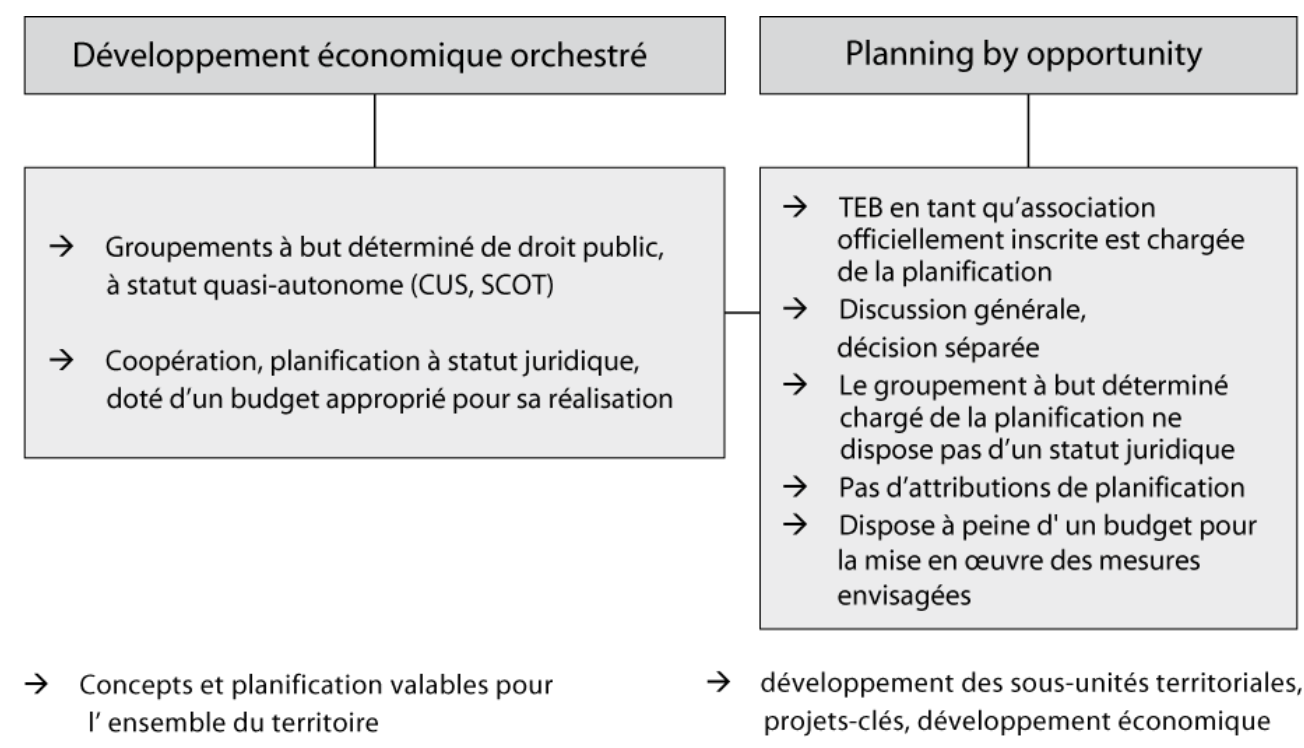

Dans ce contexte, il est réjouissant de constater que les erreurs du passé ont entre-temps été débattues publiquement et montrées du doigt, ce qui permet déjà de poser des jalons pour envisager le futur de la région. Il est également réjouissant de constater que des initiatives telles que «Metrobasel» encouragent de nouveaux développements et de nouvelles façons de penser. Tout comme il est heureux qu'une réforme administrative ait mis en place un "Stadtpräsidialamt » doté d'une plus grande responsabilité propre en matière de développement urbain et métropolitain. Un changement de paradigme semble pourtant indispensable dans le domaine de la coopération trinationale pour que le développement de la région métropolitaine transfrontalière de Bâle aboutisse à une nouvelle forme institutionnelle, qu'il reste à définir. Un regard sur d'autres cas d'études peut offrir certaines orientations pour le futur. Il faut pour cela le courage de penser et d'agir de manière renouvelée. L'élan actuel dans la région trinationale de Bâle fait suite à la vision de ceux qui ont initié la coopération transfrontalière depuis des décennies. Cette opportunité doit être encore mise à profit de manière accentuée. 


\section{BIBLIOGRAPHIE}

BAK-Basel Economics (2005). - Die Stärken und Schwächen des Life Sciences Standortes Schweiz im globalen Wettbewerb, Conférence du 8 juin 2005, Bâle, en collaboration avec les cantons de BâleVille et Bâle-Campagne.

Basler Zeitung (3 janvier 2008). - Im Baselbiet regiert der Eigensinn. Gemeindefusionen erhöhen die Effizienz - doch für die Regierungen sind sie kein Thema, p.19.

Basler Zeitung (3 janvier 2008). - Politiker fordern Fusionen, p.1.

de Spindler J. (1998). - FOCJ - Ein Konzept zur Neuordnung der Zusammenarbeit öffentlichrechtlicher Gebietskörperschaften, Berne.

Eder S., Sandtner M. (2000). - « Staatsgrenzen in der TriRhena - Barriere oder Stimulus? », Regio Basiliensis, ${ }^{\circ} 41 / 1$, p.15-26.

Fichtner U. (2006). - « Architekturen grenzübergreifender Kooperation und raumbezogene Identität am Oberrhein », Europa Regional, n¹4/2003, p.102-117.

Frey B.S., Eichenberger R. (1999). - The New Democratic Federalism for Europe, Cheltenham et Northampton.

Füeg R. (2006). - Wirtschaftsstudie Nordwestschweiz.

Gallusser W. (éd.) (1994). - Political Boundaries and Coexistence, Proceedings of the IGU-Symposium, Bâle.

Gschwind E. (2003). - Schrittmacherin oder Bremsklotz? Die Rolle der Politik bei der Schaffung des grenzüberschreitenden Wirtschaftsraums Regio TriRhena, Travail de diplôme, Master of Advanced European Studies MAES, Europainstitut, Bâle, 65p.

Kirchgessner G., Feld L.P., Savioz M.R. (1999). - Die direkte Demokratie: Modern, erfolgreich, entwicklungs und exportfähig, Bâle, Genève, Munich, Helbing \& Liechtenhand / Franz Wahlen.

Minghi J.V. (1977). - « Grenzen in der politischen Geographie », in Matznetter J. (éd.). -Politische Geographie, Darmstadt, Wege der Forschung, pp. 338-389.

Newman D., Paasi A. (1998). - « Fences and neighbors in the postmodern world: Boundary narratives in political geography ", Progress in Human Geography, n²2, p.186-207.

Regionalkonferenz der Regierungen der Nordwestschweiz (2005). - Wirtschaftsraum Nordwestschweiz, Conférence régionale des gouvernements de la Suisse du Nord-Ouest, 10 juin 2005.

Saladin P. (1995). - Wozu noch Staaten? Zu den Funktionen eines modernen demokratischen Rechtsstaats in einer zunehmend überstaatlichen Welt, Berne, Munich.

Schneider-Sliwa R. (2003). - Regio TriRhena und südlicher Oberrhein: Ein Raum ohne Grenzen?, Bâle, Institut de Géographie.

Speiser B. (1993). - Europa am Oberrhein: der grenzüberschreitende Regionalismus am Beispiel der oberrheinischen Kooperation, Travail de diplôme, Université de St. Gall Bâle, 276p. 
Weber-Mandrin M. (2001). - Öffentliche Aufgaben der Kantonsverfassungen. Zürcher Studien zum

öfentlichen Recht, Zurich

Wirtschaftskammer Baselland (2004). - Die EU-Osterweiterung aus Sicht der Schweiz, Liestal.

Sources internet :

www.alsace-biovalley.com

www.biovalley.com

www.alsace.cnrs.fr; www.inserm.fr/fr/home.html

www.regbas.ch/d_meilensteine.cfm

www.regiotrirhena.org/dn_verein_regiotrirhena/

www.srl.de/dateien/dokumente/de/trinationale_agglomeration_basel_tab.pdf

www.srl.de/dateien/dokumente/de/trinationale_agglomeration_basel_tab.pdf

http://archiv.jura.uni-saarland.de/BIJUS/karlsruhe/gesetz.htm

Konferenz der Kantonsregierungen 2007, www.kdk.ch/int/kdk/de/triagglo.html

Etat des sources internet mobilisées au 3 janvier 2008.

\section{RÉSUMÉS}

Dans un monde supposément intégré, les frontières politiques constituent toujours un élément ségrégant, un résumé de la construction humaine de l'espace et un lieu où les sociétés humaines et les Etats se disputent l'espace. Les espaces frontaliers constituent des lignes de démarcation entre différentes normes juridiques qui réunissent des cultures de planification différentes, en particulier dans les agglomérations transfrontalières. Ces dernières nécessitent de mettre en œuvre des politiques de planification et d'aménagement transfrontalières spécifiques à l'échelle métropolitaine. Ces espaces nécessitent également de prendre en compte la manière dont les structures culturelles et historiques se sont développées au cours du temps. A la recherche de modèles institutionnels permettant d'assurer le développement métropolitain, les pays limitrophes du sud du Rhin supérieur ont initié des schémas de développement relatifs à la planification métropolitaine. Leurs modèles institutionnels ont été construits selon différentes priorités et différentes compétences en matière de souveraineté. L'adoption simple de modèles de développement métropolitain ayant faits leurs preuves ailleurs est donc limitée. La contribution traite des modèles de développement métropolitains de l'agglomération de Strasbourg et de la région de Bâle et remet en question l'approche adoptée par l'Eurodistrict bâlois, dans laquelle une structure de développement a été adoptée sans au préalable fonder les bases d'une planification institutionnelle.

Political borders still divide in a world geared at integration; they are an abstract human construct of space and often reason why human society and states are in conflict. Separating different legal standards and planning cultures, border areas, and crossborder agglomerations in particular, are sensitive areas in metropolitan development. These areas require specific transboundary development and planning and a particular understanding of specific cultural and historically-embedded structures. In search of institutional models for metropolitan development, the countries bordering the southern Upper Rhine have paved their own path of development, differing in the emphasis and power given to their institutional models for urban planning to exercise public authority. A simple transfer of successful models of metropolitan 
development thus has limitations. This article discusses models of metropolitan development in the larger area of Strasbourg and Basel. In particular, it questions the decision made by Basel to adopt an Eurodistrict approach without ensuring that its basic structure provides for institutionalised metropolitan development.

Politische Grenzen sind auch in einer auf Integration bedachten Welt noch ein trennendes Element, ein abstraktes menschliches Raumkonstrukt und ein Ort der Auseinandersetzung der menschlichen Gesellschaft und des Staates mit dem Raum. Als Trennlinie unterschiedlicher rechtlicher Normen und Planungskulturen stellen Grenzräume, insbesondere grenzübergreifende Agglomerationen auch Problemgebiete für Metropolitanentwicklung dar, die der besonderen ?grenzüberschreitenden? Planungs- und Raumordnungspolitik bedürfen, aber auch eines besonderen Verständnisses für die spezifischen kulturellen und historischgewachsenen Strukturen. Auf der Suche nach institutionellen Modellen für die Metropolitanentwicklung haben die Anrainerländer am Südlichen Oberrhein eigene Entwicklungspfade beschritten und ihren institutionellen Modellen für die Metropolitanplanung unterschiedliche Gewichtung und hoheitliche Kompetenzen gegeben. Die einfache Übernahme von erfolgreichen Modellen der Metropolitanentwicklung ist daher nur begrenzt möglich. Der Beitrag behandelt die Modelle der Metropolitanentwicklung des Grossraums Strasbourg und der Region Basel und hinterfragt den Eurodistrikt-Ansatz Basels, in dem ein Gefäss für die Entwicklung übernommen wurde, ohne wichtige Grundstrukturen einer institutionalisierten Metropolitanplanung zu besitzen.

\section{INDEX}

Mots-clés : coopération transfrontalière, Eurodistrict Trinational, régions métropolitaines, Rhin supérieur

Keywords : cross-border cooperation, metropolitan regions, Trinational Eurodistrict Basel, Upper Rhine Valley, urban gouvernance

Schlüsselwörter : Gouvernanz, grenzüberschreitende Kooperation, Metropolitanregionen, Oberrhein, Trinationaler Eurodistrict Basel

\section{AUTEUR}

\section{RITA SCHNEIDER-SLIWA}

Departement Umweltwissenschaften, Geographie/Stadt- und Regionalforschung, Universität Basel, Klingelbergstr. 27, CH-4056 Bâle/Suisse. - Rita.Schneider-Sliwa@unibas.ch www.humgeo.unibas.ch 